\title{
Der Begriff der Arbeit bei Hitler
}

Hitlers Weltanschauung ist vielfach untersucht worden. ${ }^{1}$ Aber eigentümlicher Weise spielt der Begriff der Arbeit keine bedeutsame Rolle, wenn man von Reflektionen über den Namen der Nationalsozialistischen Arbeiterpartei und der Frage, ob die NSDAP eine Politik für Arbeiter gemacht hat, absieht. Diese Leerstelle ist umso seltsamer, als Arbeit für den Nationalsozialismus ein zentraler Begriff war und deshalb nicht unwichtig ist, was Hitler dazu gesprochen oder geschrieben hat. Daher soll im Folgenden der Versuch unternommen werden, eben nach Hitlers Vorstellung von Arbeit in seinen Reden und Schriften zu forschen, zumal er in einer frühen grundlegenden Rede 1920 Antisemitismus und Arbeit miteinander weltanschaulich verschränkt hat. Die Analyse dieser Rede steht am Anfang des Aufsatzes (I) und wird anschließend in ihren ideologischvölkischen Kontext gestellt (II), der nicht bloß reaktionär war, sondern mit seiner Kapitalismuskritik im Zusammenhang mit der modernen Gesellschaft betrachtet werden muss (III). Nach der Schilderung, wie Arbeit in Hitlers Wahlagitation vor 1933 erscheint (IV), steht der 1. Mai 1933 als zentrales Moment von Arbeits- und Volksgemeinschaftsrhetorik im Mittelpunkt (V). Beide Seiten von Arbeit im Nationalsozialismus: Dienst an der Volksgemeinschaft (VI) und Zwang und Gewalt (VII) erscheinen auch in Hitlers Äußerungen nach 1933 klar und deutlich. Der rassistisch begründete Arbeitsbegriff führt zu einem entgrenzten Utilitarismus, der massenmörderische Dimension annahm: „Vernichtung durch Arbeit“.

\section{Arbeit und Antisemitismus}

Einer seiner ersten großen programmatischen Reden hielt Adolf Hitler am 13. August 1920 im Festsaal des Münchner Hofbräuhauses zum Thema „Warum sind wir Antisemiten?“. ${ }^{2}$ In dieser Rede spielte Arbeit eine zentrale Rolle. Mit diesem Begriff entfaltete Hitler seine Weltanschauung von der rassistischen Superiorität der „Arier“ wie der Vernichtung der „Juden“. Während Arbeit als

1 Siehe insb.: Eberhard Jäckel, Hitlers Weltanschauung. Entwurf einer Herrschaft, erw. u. überarb. Neuausg. Stuttgart 1981.

2 Zit. n.: Hitler. Sämtliche Aufzeichnungen 1905-1924, hrsg. v. Eberhard Jäckel zusammen mit Axel Kuhn, Stuttgart 1980, S. 184-204. Erstmals abgedruckt in: Reginald H. Phelps, Hitlers „grundlegende“ Rede über den Antisemitismus, in: VfZ 16 (1968) 4, S. 390-420. 
instinktmäßiger Selbsterhaltungstrieb noch Menschen wie Tieren gemeinsam gewesen sei, habe sich bei den Menschen eine zweite Stufe entwickelt, die Arbeit aus reinem Egoismus. Auch diese Stufe sei überwunden worden zugunsten der Arbeit aus „sittlich-moralischem Pflichtgefühl“, einer Tätigkeit, „die ich nicht um meiner selbst willen ausübe, sondern auch zu Gunsten meiner Mitmenschen“.

Hitler gewann seinen Begriff der Arbeit aus dem Entwurf einer Geschichte der „nordischen Rassen“, die, weil sie im Norden der Welt gelebt hätten, „in jenen unerhörten Eiswüsten, in jenen Stätten, die nur das kärglichste Dasein boten“, gezwungen gewesen wären, nicht nur für den Einzelnen, sondern für die ganze Sippe zu arbeiten. Während im Süden den Menschen das Lebensnotwendige nahezu ohne Arbeit im überreichen Maße geboten worden wäre, hätten die „nordischen Menschen“ um ihre Existenz kämpfen müssen. „Dieser Norden zwang die Menschen zu weiterer Tätigkeit, zur Bekleidung, zum Bau eigener Behausungen, Höhlen, später Wohnungen, kurz, er hat ein Prinzip praktisch geboren, das Prinzip der Arbeit.“

Der Kampf ums Dasein hätte darüber hinaus zu „Rassereinzucht“ geführt. „Was schwächlich und kränklich war“, so Hitler, „konnte diese fürchterliche Periode nicht überstehen, sondern sank frühzeitig ins Grab und über blieb ein Geschlecht von Riesen an Kraft und Gesundheit.“ In ihrem äußeren Wirkungskreis begrenzt, hätten sich diese Menschen mehr und mehr den inneren Dingen zugewandt.

Diese drei Errungenschaften: das erkannte Prinzip der Arbeit als Pflicht, die Notwendigkeit, nicht nur für den Einzelnen und aus Egoismus, sondern zum Bestande dieser ganzen, wenn auch oft nur ganz kleinen Masse von Menschen, dieser kleinen Sippen, zweitens die unbedingte körperliche Gesundheit und dadurch die geistige normale Gesundheit, und drittens das tief-innerliche Seelenleben hat diesen nordischen Rassen die Möglichkeit gegeben, staatenbildend über die übrige Welt zu ziehen.

Die Fähigkeit zur Staatenbildung und zu einer blühenden Kultur läge, so unterstrich Hitler, ausschließlich in der Auffassung des Begriffs Arbeit begründet. Arbeit sei nicht als Zwang, sondern als „notgeborene Notwendigkeit“ verstanden worden; die unwirtlichen Lebensumstände erforderten gemeinsames Arbeiten und Arbeitsteilung. Arbeit sei, so Hitlers Quintessenz, von den „nordischen Rassen“ als „soziale Pflicht“ angesehen worden.

Ganz anders die „Juden“. Zynisch zitiert Hitler Gottes Fluch aus dem ersten Buch Mose nach der selbst verschuldeten Vertreibung von Adam und Eva aus dem Paradies: „Im Schweiße deines Angesichts sollst du dein Brot essen“, um als sei dieser Satz nicht auch Teil der christlichen Bibel - den Zuhörern zu erklären, dass für die Juden demnach Arbeit als Strafe gelte. „Meine verehrten Anwe- 
senden! Hier trennt uns schon eine ganze Welt; denn wir können Arbeit nicht als Strafe auffassen, weil wir sonst alle Sträflinge wären.“ Den Juden, so Hitler, sei Arbeit nicht sittliche Pflicht, sondern höchstens Mittel zur Selbsterhaltung. So verstanden könnte aber jede Tätigkeit, die ohne Rücksicht auf die Mitmenschen dazu dient, sich zu ernähren, also zum Beispiel auch das Ausrauben von Karawanen oder die Ausplünderung verschuldeter Bauern, als Arbeit gekennzeichnet werden. Juden, die sich nicht wie die nordischen Rassen „reingezüchtet“ hätten, seien nicht in der Lage, Staaten zu bilden, sondern könnten nur als „Parasiten am Körper anderer Völker“ existieren.

Damit lägen die Unterschiede zwischen den beiden „Rassen“ offen zutage: „Ariertum bedeutet sittliche Auffassung der Arbeit und dadurch das, was wir heute so oft im Munde führen: Sozialismus, Gemeinsinn, Gemeinnutz geht vor Eigennutz - Judentum bedeutet egoistische Auffassung der Arbeit und dadurch Mammonismus und Materialismus, das konträre Gegenteil von Sozialismus. Und in dieser Eigenschaft, über die er nicht hinaus kann, die in seinem Blut liegt, er selbst erkennt das an, in dieser Eigenschaft allein schon liegt die Notwendigkeit für den Juden, unbedingt staatenzerstörend auftreten zu müssen. Er kann nicht anders, ob er will oder nicht.“

In dieser Passage lässt sich ein zentrales Element des nationalsozialistischen Antisemitismus erkennen: Es gibt für Juden kein Entrinnen. Sie mögen als Einzelne gut oder böse sein, fleißig oder faul, anpassungsbereit oder eigensinnig, es hilft ihnen nichts. Die Forderung nach restloser Assimilation der Juden seitens der älteren Generation der Antisemiten war für Hitler obsolet, im Gegenteil, sie verschleierte nur den Blick für das unveränderliche jüdische „Rassenschicksal“. Dagegen helfe, so Hitler, keine Aufklärung, kein bloß wirtschaftlicher Kampf, sondern nur die Tat und die Organisation der Massen.

Darüber waren wir uns klar, wenn diese Bewegung nicht in die breiten Massen dringt, sie organisiert, dann ist alles vergeblich, dann wird es nie gelingen, unser Volk zu befreien, und wir werden nie daran denken können, unser Vaterland von neuem aufzubauen. Niemals kann hier die Erlösung kommen von oben, sie kann und wird nur kommen von der breiten Masse, von unten empor.

Die notwendigen sozialen Reformen zugunsten derer, „die Tag für Tag für die Volksgemeinschaft arbeiten“, müssen einhergehen mit dem

Kampf gegen den Gegner jeder sozialen Einrichtung: das Judentum. Auch hier wissen wir genau, dass die wissenschaftliche Erkenntnis bloß die Vorarbeit sein kann, dass aber hinter dieser Erkenntnis die Organisation kommen muss, die einst zur Tat übergeht, und die Tat bleibt bei uns unverrückbar fest, sie heißt: Entfernung der Juden aus unserem Volke. 
Das Polizeiprotokoll vermerkt an dieser Stelle stürmischen, lang anhaltenden Beifall und Händeklatschen.

\section{Völkische Traditionen}

Hitlers rassistisches, antisemitisches Arbeitskonzept war nicht seine eigene originäre Leistung, sondern stand in einer langen Tradition völkischen Denkens. ${ }^{3}$ Einer der wichtigsten deutschen politischen Publizisten am Beginn des 19. Jahrhunderts, Paul Ferdinand Friedrich Buchholz, machte in seinem 1803 erschienenen Büchlein „Moses und Jesus oder über das intellektuelle und moralische Verhältniß der Juden und Christen“ unmißverständlich klar: Für einen Angehörigen eines christlichen Volkes sei Arbeit „das Mittel, wodurch er seinen Standort in der Gesellschaft behauptet. Er fühlt sich nicht als Individuum, sondern als Fragment eines kleinere oder größeren Ganzen“, dessen Bestreben „immer“ dahin gehe, „sich anderen nützlich zu machen, indem diese ihm wieder nützlich werden.“ Juden hingegen nähmen nicht an der „Nazionalarbeit“ teil. ${ }^{4}$

In Gustav Freytags Roman Soll und Haben (1855) steht die „Arbeit auf deutsche Weise“, die sich dem Gemeinwohl der Nation verpflichtet fühlt, der egoistischen, betrügerischen, gewinnorientierten Wirtschaftstätigkeit der jüdischen Romanfigur Itzig Veitel gegenüber. ${ }^{5}$ Explizit entwickelte der Volkskundler und Publizist Wilhelm Heinrich Riehl 1861 in seinem Buch „Die deutsche Arbeit“ eine Vorstellung von einer spezifisch nationalen Form des Arbeitens. ${ }^{6}$ Ähnlich

3 Zur rassistischen Konnotation des nationalsozialistischen Arbeitsbegriffs siehe auch: Rüdiger Hachtmann, Vom „Geist der Volksgemeinschaft durchpulst“- Arbeit, Arbeiter und die Sprachpolitik der Nationalsozialisten, in: Zeitgeschichte-Online, Themenschwerpunkt „Arbeit“ [http://www.zeitgeschichte-online.de/themen/vom-geist-der-volksgemeinschaft-durchpulst], eingesehen 24.6.2014.

4 Friedrich Buchholz, Moses und Jesus oder über das intellektuelle und moralische Verhältniß der Juden und Christen, Berlin 1803, S. 82, zit. n.: Klaus Holz/Jan Weyand, Arbeit und Nation. Die Ethik nationaler Arbeit und ihre Feinde am Beispiel Hitlers (unveröff. Paper, ich danke beiden Autoren für die Einsichtnahme). Zu Buchholz siehe: Deutsche Biographische Enzyklopädie, hrsg. v. Walter Killy, Bd. I, München et al. 1995, S. 184.

5 Vgl. dazu die Aufsätze von Christine Achinger, Antisemitismus und „Deutsche Arbeit“. Zur Selbstzerstörung des Liberalismus bei Gustav Freytag, in: Nicolas Berg (Hrsg.), Kapitalismusdebatten um 1900. Über antisemitisierende Semantiken des Jüdischen, Leipzig 2011, S. 361-388, sowie: Heinrich Schwendemann, Gustav Freytags Soll und Haben (1855) - Wegbereiter des ökonomischen Antisemitismus, in: ebd., S. 333-360.

6 Wilhelm Heinrich Riehl, Die Deutsche Arbeit, Stuttgart 1861; vgl. dazu: Joan Campbell, Joy in Work, German Work: The National Debate, 1800-1945, Princeton/New Jersey 1989, S. 32-46. Pina Bock (Leipzig) arbeitet an einer Dissertation zum Topos „Deutsche Arbeit“ im 19. Jahrhundert. 
wie bei Freytag war Arbeit das Charakteristikum der Deutschen. Wieder zeichnete sich „deutsche Arbeit“ durch ihre Gemeinnützigkeit aus. Das deutsche Volk, so Riehl, denkt, wenn es von Arbeit spricht, ,an eine aus sittlichen Motiven entspringende, nach sittlichem Ziele ringende That, die mit dem Nutzen für uns selbst zugleich den Nutzen für andere Leute verbindet" ${ }^{\text {"7 }}$. Diese Auffassung von Arbeit unterscheide sich scharf vom „Schaffen und Raffen blos um eigennützigen Gewinns willen“8, wofür selbstredend vor allem die „Juden“ stehen, deren Arbeit Handel und „Schacher“ seien. Allerdings gab es - im Gegensatz zum eliminatorischen Antisemitismus Hitlers - im Denken Riehls einen Ausweg für die jüdische Minderheit:

\footnotetext{
Man machte sie deutsch, indem man ihnen die nationalen Formen unserer Arbeit erschloß und damit zugleich deutsche Gedanken von Arbeitsehre und Arbeitsmoral einpflanzte. Der Jude hinterm Pfluge und in der Werkstatt verliert sein semitisches und mittelaltriges Volksgepräge, weil er die theils angestammten, theils aufgezwungenen Schranken seiner Arbeit fallen lässt. ${ }^{9}$
}

Ebenso war die Vorstellung vom „schaffenden Deutschen“ und „raffenden Juden“ fester Bestandteil völkischen Denkens. Nach dem Börsenkrach 1873, in dem viele Kleinanleger ihr Vermögen verloren hatten, veröffentlichte Otto Glagau in der weitverbreiteten Zeitschrift Die Gartenlaube eine Artikelserie, in der er den Beruf des Börsenmaklers als betrügerisch denunzierte und das Finanzkapital als ,jüdisch“. In seiner Streitschrift Deutsches Handwerk und historisches Bürgertum (1879) ging er noch einen Schritt weiter und machte „die Juden“ für die Veränderungen im Wirtschaftsleben durch den Kapitalismus insgesamt verantwortlich: „Die soziale Frage“, so Glagau, „ist die Judenfrage!“10 Dieser Identifizierung des Kapitalismus als ,jüdisch“ schloss sich auch der renommierte wilhelminische Historiker Heinrich von Treitschke an, der mit seiner Losung „Die Juden sind unser Unglück!“ bis in den Nationalsozialismus hinein wirken sollte. Treitschke begründete seinen Antisemitismus ökonomisch mit dem angeblichen materiellen Gewinnstreben des Juden, „der jede Arbeit nur noch als Geschäft betrachtet und die alte gemüthliche Arbeitsfreudigkeit unseres Volkes zu ersticken droht“"11.

7 Riehl, Arbeit, S. 5.

8 Ebd., S. 6.

9 Ebd., S. 64.

10 Zit. n.: Shulamit Volkov, Antisemitismus als kultureller Code. 10 Essays, München 2000, S. 29.

11 Zit. n.: Holger Schatz/Andrea Woeldike, Freiheit und Wahn deutscher Arbeit. Zur historischen Aktualität einer folgenreichen antisemitischen Projektion, Hamburg/Münster 2001, S. 51. 
Die Identifizierung des Kapitalismus als „jüdisch“ durchzog die gesamte völkische Agitation. Kapitalismus sei „praktischer Mosaismus“, schrieb der antisemitische Publizist Theodor Fritsch in seinem „Handbuch zu Judenfrage“, das, 1887 zuerst erschienen, bis 194449 Auflagen erlebte. ${ }^{12}$ Und der Ökonom Werner Sombart formuliert in seiner 1911 erschienenen Schrift „Die Juden und das Wirtschaftsleben“, dass der Jude der „reine Geschäftsmann“ sei, „der im Geist echt kapitalistischer Wirtschaft allen naturalen Zwecken gegenüber den Primat des Erwerbszwecks anerkennt““.13 Neben dem Sozialismus ließ sich auch der Antisemitismus als Weltanschauung mit den Erfahrungen der tief in den Alltag einschneidenden Veränderungen durch Industrie und Kapitalismus verbinden und zugleich zu dessen kritischer Deutung benutzen. Gerade jene Mittelschicht aus Handwerkern, Kaufleuten und kleinen Gewerbetreibenden, die sich durch die Industrieproduktion, neue urbane Distributionsformen wie Warenhäuser und veränderte Kredit- und Kapitalmärkte in ihrer Existenz bedroht fühlten, waren für die antisemitische Interpretation des rasanten gesellschaftlichen Wandels ebenso anfällig wie die ländliche Bevölkerung, deren bisherige agrarische Produktions- und Lebensweise von kapitalistischer Modernisierung, Urbanisierung und dem Weltmarkt nachhaltig verändert wurde.

Nicht zufällig war es vor allem der alte Mittelstand der Handwerker und Kleinhändler, die im Zentrum völkischer Politik standen, da diese Schichten sich in besonderer Weise von der modernen Entwicklung bedroht fühlten. Die Einführung der Gewerbefreiheit, 1869 im Norddeutschen Bund, 1872 dann im ganzen Deutschen Reich, brach die Macht der Zünfte. Während sich die Handwerker in den folgenden Jahren mit dem Befähigungsnachweis und der Einrichtung von Handwerkskammern manches zünftige Privileg zurückerobern konnten, waren die Kleinhändler desolat organisiert und gegenüber den ökonomisch erforderlichen Modernisierungen zumeist machtlos. Konsumvereine, Versandhäuser und in den Städten Warenhäuser füllten die Versorgungslücken. ${ }^{14}$

12 Theodor Fritsch, Handbuch der Judenfrage, 28. Aufl. 1919, S. 498, zit. n.: Heike Hoffmann, Völkische Kapitalismus-Kritik: Das Beispiel Warenhaus, in: Handbuch zur „Völkischen Bewegung“ 1871-1918, hrsg. v. Uwe Puschner, Walter Schmitz u. Justus H. Ulbricht, München et al. 1996, S. 558-571, hier S. 559.

13 Werner Sombart, Die Juden und das Wirtschaftsleben, Leipzig 1911, S. 155, zit. n.: Hoffmann, Völkische Kapitalismus-Kritik, S. 559.

14 Heinz-Gerhard Haupt (Hrsg.), Die radikale Mitte. Lebensweise und Politik von Handwerkern und Kleinhändlern in Deutschland seit 1848, München 1985; ders., „Bourgeois und Volk zugleich?“ Zur Geschichte des Kleinbürgertums im 19. und 20. Jahrhundert, Frankfurt a.M./ New York 1978. 
Dementsprechend engagierten sich Antisemiten in den Mittelstandsvereinigungen und mittelständische Verbände vertraten antisemitische Positionen. Dabei reichte die Vorstellung, wer zum Mittelstand gehöre, sehr weit. Nicht nur Handwerker und Kaufleute sollten dazu zählen, sondern laut der Rede von Theodor Fritsch auf der Gründungsversammlung der sächsischen Mittelstandsvereinigung 1905 ,

alle Stände, die durch redlichen Erwerb ihr Dasein fristen: den Arzt, den Lehrer, den Richter, den Beamten, den Gelehrten, den Künstler, den Techniker, den Fabrikanten, den Bauer, den Gutsbesitzer, den Hausbesitzer, den sittlich denken Arbeiter, kurz all diejenigen, die [...] in der Mitte stehen zwischen dem Großkapitalisten und dem Tagelöhner ${ }^{15}$.

Daran klang schon jene Einheit „aller schaffenden Stände“ an, die eine Grundlage für das Konzept der Volksgemeinschaft bilden sollte.

\title{
3 Kapitalismuskritik
}

So rückwärts gewandt die antisemitische, Handwerk und Bauerntum verklärende Rhetorik anmutet, so war sie doch eine „moderne“ Antwort auf die unumkehrbaren Transformationen der Gesellschaft. ${ }^{16}$ Die Differenzierung von „schaffendem“ und „raffendem“ Kapital bezog sich nicht auf eine vormoderne Wirtschaftsordnung als vielmehr auf den Kapitalismus; der so genannte nationale Sozialismus ging von der sozialen Existenz der Arbeiterschaft als neue, durch die kapitalistische Industrie geschaffene Klasse aus und strebte deren Integration in eine völkisch strukturierte Gesellschaft an. Der Antisemitismus ermöglichte eine Kapitalismuskritik, ohne den Kapitalismus abzuschaffen.

Hitler wehrte sich in seiner Rede 1920 gegen den Vorwurf, die Nationalsozialisten bekämpften nur das Börsen- und Leihkapital, nicht aber das Industriekapital überhaupt, indem er dieses als Werkzeug definierte.

\begin{abstract}
Was heißt Industriekapital? Es ist ein in der Größe sich allmählich verändernder Faktor, nur ein relativer Begriff. Es stellte einst vor: die Nadel, der Zwirn, die Werkstätte und vielleicht die paar Groschen Geldes, die der Schneidermeister in Nürnberg besaß im 13. Jahrhundert. Es war dies die Summe dessen, was er zur Arbeit nötig hatte, d. h. Werkzeug, Werkstätte und eine gewisse Summe, um ihm überhaupt eine gewisse Zeit das Leben zu ermöglichen.
\end{abstract}

15 Theodor Fritsch, Mittelstand, Kapital-Herrschaft, Monarchie, Leipzig 1906, S. 18, zit. n.: Hoffmann, Völkische Kapitalismus-Kritik, S. 559.

16 Immer noch anregend: Jeffrey Herf, Reactionary Modernism. Technology Culture and Politics in Weimar and the Third Reich, Cambridge 1984. 
Mit der Zeit sei aus der Werkstätte eine Fabrik geworden, aus dem Webstuhl eine Maschine. „Aber Werkstätte und Werkzeug, Maschine und Fabrik an und für sich sind kein Wert, der von sich selber Wert erzeugt, sondern nur Mittel zum Zweck, wird erst Wert erzeugend, wenn mit ihnen gearbeitet wird. Das werterzeugende ist die Arbeit. “17 Dem nationalen Staat und Volk verpflichteten Industriekapital stehe das internationale Finanzkapital gegenüber, das, organisiert von Juden, nationale Ökonomien zerstöre. Diese Sichtweise, dass allein die Arbeit die Grundlage alle Werte sei, blieb eine Leitlinie in Hitlers Denken. 1937 unterstrich er auf einer Massenkundgebung in Regensburg:

Wir haben das Geld seines phantomhaften Charakters entkleidet und es in die Rolle gebracht, die ihm zukommt: Nicht Gold und nicht Devisenbestände, sondern die Arbeit allein ist die Grundlage des Geldes! Es gibt keine Steigerung des Lohns, wenn nicht eine Steigerung der Produktion Hand in Hand damit geht. ${ }^{18}$

Mit der Auffassung, dass Arbeit Wert schaffe, stand Hitler ganz in der Tradition der von Adam Smith begründeten Nationalökonomie des 19. Jahrhunderts, in der Arbeit als Quelle gesellschaftlichen Reichtums zur zentralen Kategorie avancierte. ${ }^{19}$ Arbeit hat - so hat Jürgen Kocka formuliert - in der bürgerlichen Epoche in Europa eine „geradezu emphatische Aufwertung [...] als Quelle von Eigentum, Reichtum und Zivilität bzw. als Kern menschlicher Existenz und Selbstverwirklichung“20 erfahren. „Arbeit ist des Bürgers Zierde, Segen seiner Mühe Preis. Ehrt den König seine Würde, ehret uns der Hände Fleiß“, dichtete Friedrich Schiller in der „Glocke“.

Gleichermaßen gründet die Marxsche Theorie auf dem wertschöpfenden Charakter der Arbeit, wobei zugleich durch die Kategorien von Gebrauchs- und Tauschwert und deren unterschiedlicher Aneignung in der kapitalistischen Gesellschaft mit Marx auch der Begriff der Entfremdung bedeutsam wird. Denn auch Marxisten haben neben der kühlen Analyse des freien Lohnarbeiters, dessen produzierter Mehrwert sich der Kapitalist unrechtmäßig aneignet, einen utopischen Begriff von nicht entfremdeter, ganzheitlicher Arbeit, die in der kommunistischen Gesellschaft individuelle Freiheit wie soziale Verkehrsform darstellt.

17 Hitler. Sämtliche Aufzeichnungen, S. 193.

18 Max Domarus, Hitler. Reden und Proklamationen 1932-1945. Kommentiert von einem Zeitgenossen, Teil I Triumph, Bd. II.: 1935-1938, Leonberg 1988, S. 698.

19 Manfred Füllsack, Arbeit, Wien 2009, S. 50-53.

20 Jürgen Kocka, Mehr Last als Lust. Arbeit und Arbeitsgesellschaft in der europäischen Geschichte, in: ders., Arbeiten an der Geschichte. Gesellschaftlicher Wandel im 19. und 20. Jahrhundert, Göttingen 2011, S. 203-224, hier S. 206. 
Die Sozialdemokratische Partei formulierte in ihrem Gothaer Programm 1875:

Die Arbeit ist die Quelle allen Reichtums und aller Kultur, und da allgemein nutzbringende Arbeit nur durch die Gesellschaft möglich ist, so gehört der Gesellschaft, d. h. allen ihren Gliedern, das gesamte Arbeitsprodukt, bei allgemeiner Arbeitspflicht, nach gleichem Recht, jedem nach seinen vernunftgemäßen Bedürfnissen. ${ }^{21}$

Die Auffassung, dass nur derjenige, der arbeitet, ein nützliches Glied der Gesellschaft sei, die sich bei den Sozialdemokraten vor allem gegen Großgrundbesitzer und Kapitalisten richtete, ließ sich durchaus antisemitisch drehen, indem die Müßiggänger und Ausbeuter als jüdisch identifiziert und die Schaffenden als nationale Gemeinschaft bestimmt wurden. Die Forderung nach „Ehre der Arbeit“, nach Aufwertung der Arbeiter als wertschaffender Kraft der Nation und nach Anerkennung von „deutscher Qualitätsarbeit“ konnte auf der Linken wie der Rechten erhoben werden..$^{22}$ Gleichermaßen konnte die Verurteilung von Nichtstun als „Faulheit“, die Verankerung von Arbeit als Pflicht und die Messung des sozialen Werts von Menschen an ihrer Arbeitsfähigkeit Personen rechter wie linker politischer Orientierung vereinen.

Mit der Priorität der Gemeinnützigkeit der Arbeit rückte das Charakteristikum der Lohnarbeit, die Bezogenheit auf den Markt und die Entlohnung in Geld, in den Hintergrund. Auch für die Nationalsozialisten galt es, in ihren Augen berechtigte materielle Ansprüche auf angemessenen Lohn anzuerkennen. In seinem Buch „Mein Kampf“ widmete Hitler der Gewerkschaftsfrage ein ganzes Kapitel. Gewerkschaften seien notwendig,

wenn durch sie soziale Ungerechtigkeiten, die in der Folge zu schweren Schädigungen des ganzen Gemeinschaftswesens eines Volkes führen müssen, verhindert werden können [... und] solange es unter den Unternehmern Menschen gibt, die von sich aus nicht nur kein Gefühl für soziale Pflichten, sondern nicht einmal für primitivste menschliche Rechte besitzen. ${ }^{23}$

Zwar verschwand der Begriff der Interessensvertretung nicht ganz aus dem Text. So hielt Hitler fest,

21 Siehe dazu die bissige Kritik von Karl Marx, Randglossen zum Programm der deutschen Arbeiterpartei, in: Karl Marx/Friedrich Engels, Werke, Bd. XIX, Berlin 1978, S. 15-32.

22 Vgl. dazu wegweisend: Alf Lüdtke, „Ehre der Arbeit“: Industriearbeiter und Macht der Symbole. Zur Reichweite symbolischer Orientierungen im Nationalsozialismus, in: ders., Eigen-Sinn. Fabrikalltag, Arbeitererfahrungen und Politik vom Kaiserreich bis in den Faschismus, Hamburg 1993, S. 283-350.

23 Adolf Hitler, Mein Kampf, 349.-351. Aufl., München 1938, S. 671. 
Volksgemeinschaft bedeutet keinen Verzicht auf die Vertretung berechtigter Standesinteressen. Auseinandergehende Standes- und Berufsinteressen sind nicht gleichbedeutend mit Klassenspaltung, sondern sind selbstverständliche Folgeerscheinungen unseres wirtschaftlichen Lebens. ${ }^{24}$

Aber Hitler mühte sich sichtlich, die Tatsache unterschiedlicher Interessen unterschiedlicher sozialer Gruppen und deren nüchterne, wenngleich kompromissbereite Vertretung zugunsten einer moralischen Verpflichtung aller Beteiligten gegenüber dem Volksganzen zu ersetzen. Dass ein Großteil gesellschaftlich notwendiger Arbeit in einer modernen Industriegesellschaft Erwerbsarbeit ist, die mit Geld entlohnt wird, dessen Höhe Gegenstand durchaus gegensätzlicher Interessenkonflikte ist, minimierte Hitler, um die „Volksgemeinschaft“ als Maßstab durchzusetzen.

Hitler versuchte, das Problem mit einer Teilung des Arbeitswerts in einen materiellen und einen ideellen Wert in den Griff zu bekommen. Der materielle Wert beruhe auf dem materiellen Nutzen, den eine bestimmte Arbeit für die Gesellschaft habe, und finde seinen Ausdruck im materiellen Lohn. Da es Unterschiede in der Bewertung des materiellen Nutzens jeweiliger Tätigkeiten gebe, seien auch unterschiedliche Löhne gerechtfertigt. Im Gegensatz zur Ungleichheit in der Bewertung des materiellen Wertes sei der ideelle Wert der Arbeit gleich bemessen.

So sicher der materielle Nutzen einer Erfindung größer sein kann als der eines alltäglichen Handlangerdienstes, so sicher ist die Gesamtheit doch auf diesen kleinsten Dienst genau so angewiesen wie auf jenen größten. Sie mag materiell einen Unterschied treffen in der Bewertung des Nutzens der einzelnen Arbeit für die Gesamtheit und kann dem durch die jeweilige Entlohnung Ausdruck verleihen; sie muss aber ideell die Gleichheit aller feststellen in dem Augenblick, in dem jeder einzelne sich bemüht, auf seinem Gebiete - welches immer es auch sein mag - sein Bestes zu tun. Darauf aber hat die Wertschätzung eines Menschen zu beruhen, und nicht auf der Entlohnung. ${ }^{25}$

In dieser Konstruktion ist der Kern nationalsozialistischer Arbeitsauffassung zu erkennen. Die Frage materielle Entlohnung von geleisteter Arbeit trat zurück gegenüber einer symbolischen, öffentlichen Anerkennung von Arbeit. Damit war auch der ideologische Weg frei, all jene gesellschaftlich geleistete Arbeit, die nicht entlohnt wird, wie vor allem die Arbeit von Frauen im Haushalt und in der Familie, als gleichwertigen Dienst an der Volksgemeinschaft propagandistisch anzuerkennen, ohne die sozialreformerische Forderung aus der Frauen-

24 Ebd., S. 372f.

25 Hitler, Mein Kampf, S. 483. 
bewegung nach Bezahlung dieser Tätigkeiten zu erfüllen noch dem Drang von Frauen nach Gleichstellung und gleichem Lohn in der Erwerbsarbeit nachzugeben. Frauen besaßen in Hitlers rassistisch-maskulinem Weltbild einen klar nachgeordneten Platz - bekanntlich wehrte er sich gegen die Ausweitung von Industriearbeit von Frauen, selbst als im Krieg die Arbeitskraft von Frauen in der Rüstungsproduktion dringend gebraucht wurde. Und doch ließ sich in der hierarchisierten Ungleichheit die ideologische Gleichwertigkeit aller „,an ihrem Platz“ propagieren.

\section{Das Thema Arbeit in Hitlers Agitation vor 1933}

Mit den gleichen rassistischen Invektiven wie 1920 in München hielt Hitler auch später seine Agitationsreden wie am 15. Juli 1925 im sächsischen Zwickau vor rund 3.500 Zuhörerinnen und Zuhörern. Wieder war es die antisemitische Trennung von internationalem Finanzkapital, das von Juden kontrolliert werde, und dem nationalen Wirtschaftskapital, das aus Anlage- und Betriebskapital bestehe, mit der Hitler sein antikapitalistisch eingestelltes Publikum zu gewinnen suchte. Wieder entwickelte er am Begriff der Arbeit, dem angeblichen Unvermögen der Juden zu produktiver Arbeit einerseits und der deutschen Arbeit andererseits, die aufgebaut sei ,auf der Hingabe des einzelnen für die Gesamtheit, erst später geben die anderen ihm dann seinen Teil wieder zurück. Was er schafft, schafft er für die Allgemeinheit, ob das der Glasbläser ist, der Schmied, der Straßenfeger." ${ }^{26}$ Den Juden fehle diese kulturschöpferische Kraft:

Sehen Sie sich diesen Saal an! Wer hat diese Lampen erfunden, wer den Stoff, der den Saal schmückt? Wer ist der Erfinder? Nicht ein Jude ist unter ihnen! Und wer sind die Händler? Lauter Juden!!!27

Der Weltkrieg sei verloren worden aufgrund der Zerrissenheit des deutschen Volkes. Hitler:

Wir sind zerrissen worden durch zwei Begriffe. Der eine Begriff hieß ,Sozial', und dieser Begriff wurde falsch ausgelegt. Ein Jude war es, der diesem Begriff Falsches unterschob. Der zweite Begriff hieß ,National' und wurde auch falsch ausgelegt. Sowenig wie mit dem

26 Adolf Hitler, Rede auf der NSDAP-Versammlung in Zwickau, 15.7.1925, in: ders., Reden, Schriften, Anordnungen Februar 1925 bis Januar 1933, Bd. I, hrsg. u. komment. v. Clemens Vollnhals, München et al. 1992, S. 118-139, Zitat: S. 129.

27 Ebd, S. 130. 
Begriff ,sozial' eine bestimmte Wirtschaft zu tun hat, hat das ,national' nichts zu tun mit einer bestimmten Staatsauffassung. National ist jeder, der seinem Volke gibt, was ihm gehört. Ebenso ist sozial nur ein Mensch, der sich aufopfert für sein Volk, der sich bereit erklärt, seinem Volk zu geben, was ihm gebührt. ,Volk‘ verstanden als Volksgemeinschaft, von unten bis oben. [...] Die Stärke eines Volkes beruht nicht auf dem hochgezüchteten Genie, sondern auf der Harmonie der körperlichen und geistigen Stärke, der Harmonie von Stirne und Faust. Ohne Wiederherstellung dieses inneren Gleichgewichts aber, der Volksgemeinschaft, ist ein Wiederaufstieg unseres Volkes unmöglich. ${ }^{28}$

Vor etwa 50 Vertretern aus Industrie und Handel unterstrich Hitler im Juni 1926 in Essen, dass Kapital, Arbeit und Nation untrennbar zusammengehörten. Es sei keineswegs egal, unter welcher Kontrolle die Betriebe stünden. Wirtschaft sei mit dem Volkstum identisch, daher sei eine nationale Kontrolle absolut notwendig. Die grundsätzliche Frage für die deutsche Wirtschaft bestehe darin, ein angemessenes Verhältnis zwischen Bevölkerungszahl und Bodenfläche zu finden. Gelänge es nicht, durch Export mehr Arbeit zu schaffen, könnten bald 20 Millionen Deutsche nicht mehr ausreichend ernährt werden.

Sie bildeten die große Gefahr“, so der Bericht der Rheinisch-Westfälischen Zeitung, „nicht so sehr deswegen, weil Hungerrevolten oder Unruhen ausbrechen könnten, sondern weil sie - schlecht oder recht als Erwerbslose unterstützt - sich der Arbeit entwöhnten, zu Tagedieben würden, die das ganze Volk gesundheitlich und moralisch verseuchten. ${ }^{29}$

Immer wieder attackierte Hitler „die jüdisch-marxistischen Führer“, die die Begriffe Kapital, Eigentum, Unternehmertum verdreht hätten. Indem sie gegen das deutsche Unternehmertum und die nationale Wirtschaft kämpften, sägten sie am Ast, auf dem auch die Arbeiter selbst säßen, während sie „den eigentlichen Feind, das überstaatliche, internationale Börsen- und Leihkapital“ schonen würden. „Unser Kampf“, so Hitler auf einer Wahlkampfveranstaltung im thüringischen Eisenach im Januar 1927, „gilt den nichtschaffenden Schmarotzern, den nichtschaffenden Drohnen, unter Schutz der schaffenden Eigenpersönlichkeit und dem Eigentum.“" ${ }^{30}$

28 Ebd., S. 134.

29 Adolf Hitler, Rede vor Wirtschaftsführern in Essen, 18.6.1926, in: ders., Reden, Bd. I, S. 478-480, Zitat: S. 479. Die Zahl von 20 Millionen Deutschen, die angeblich zu viel wären, schrieb Hitler fälschlicherweise dem ehemaligen französischen Premierminister George Clemenceau zu. Stattdessen stammt die Zahl aus dem Buch „L'Allemagne ennemie“ von Jeanne und Frédéric Régamey, Paris 1913 (siehe Anm. 10, in: Adolf Hitler, Reden, Schriften, Anordnungen Februar 1925 bis Januar 1933, Bd. II/1, hrsg. u. komment. v. Bärbel Dusik, München et al. 1992, S. 447).

30 Adolf Hitler, Rede auf einer NSDAP-Versammlung in Eisenach, 13.1.1927, in: ders., Reden, Bd. II/1, S. 120-122, Zitat: S. 121. 
Auf der anderen Seite stand der Appell an die Volksgemeinschaft. „Was wir seit 1914 erlebten“, so Hitler in Hamburg am 6. September 1930 in einer Wahlkampfrede vor mehreren tausend Zuhörerinnen und Zuhörern kurz vor den Reichstagswahlen, die der NSDAP mit 18,3 Prozent der Stimmen als nunmehr zweitstärkste politische Kraft den Durchbruch bescherte,

verschwindet vor diesem neuen Erleben der echten Volksgemeinschaft, des Sichwiederfindens der Stände der Nation. Wir Nationalsozialisten erkennen als unsere größte Aufgabe, den deutschen Arbeiter zur Nation zurückzuführen, ihn als Granitblock einzufügen. Das Bürgertum aber muß aus dem Klassendünkel zur Volksgemeinschaft gebracht werden. Auf dieser Grundplattform werden wir eine neue Macht aufbauen. ${ }^{31}$

Und gleichermaßen einen Tag später in Nürnberg:

Das ist das Neue an unserer Bewegung, daß sie den Menschen langsam von der alten Überzeugung losreißt, daß die verhängnisvolle Zersplitterung aufhören muß, daß sich Stadt und Land, Arbeiter und Bauer, Angestellter und Beamter wieder eins fühlen in dem Bewußtsein, wer die Macht wirklich darstellt, das deutsche Volk in seiner Gesamtheit oder die Parteienzersplitterung. ${ }^{32}$

\section{1. Mai 1933: „Ehret die Arbeit und achtet den Arbeiter“}

Gerade der 1. Mai 1933 sollte nach dem Willen der NS-Führung zu einer großen, reichsweiten und nachhaltigen Manifestation eines Verständnisses von Arbeit als Dienst an der Volksgemeinschaft, jenseits klassenkämpferischer Interessenvertretung, und der Integration der Arbeiterschaft in den Nationalsozialismus werden. Nicht von ungefähr war es die Hitler-Regierung, die als erste deutsche Regierung den 1. Mai, den „Kampftag der Arbeiterklasse“, in einen „Tag der nationalen Arbeit“ umdefinierte und zugleich diesen Tag zu einem staatlichen Feiertag erhob. „Ehret die Arbeit und achtet den Arbeiter!“ war das offizielle Motto dieses 1. Mai 1933.

31 Adolf Hitler, Rede in Hamburg, 6.9.1930, in: ders., Reden, Schriften, Anordnungen Februar 1925 bis Januar 1933, Bd. III/3, hrsg. u. komment. v. Christian Hartmann, München u. a. 1995, S. 384-386, Zitat: S. 385.

32 Adolf Hitler, Rede in Nürnberg, 7.9.1930, in: ebd., S. 387-390, Zitat: S. 389. 
Der Tag wurde gänzlich inszeniert. ${ }^{33}$ Er begann um 9 Uhr mit einer „Kundgebung der Jugend“ im Berliner Lustgarten und Ansprache des Reichspräsidenten Hindenburg. Um die Mittagszeit empfing Hitler als Reichskanzler Arbeiterdelegationen aus dem gesamten Reichsgebiet, aus Österreich, Danzig und aus dem Saargebiet, und stellte sie anschließend dem Reichspräsidenten vor. In der Hoffnung, Handlungsspielräume zu erhalten, hatten sich die (noch) freien Gewerkschaften bereit erklärt, den „Tag der nationalen Arbeit“ zu unterstützen. Überall im Reich wurden die Gewerkschaftshäuser mit schwarz-weiß-roten Fahnen geschmückt. Auf dem Berliner Tempelhofer Feld war eine riesige Massenkundgebung, zu der zahlreiche Belegschaften Berliner Betriebe geschlossen aufmarschierten, veranstaltet worden, hinter der Rednertribüne hingen drei riesige Hakenkreuzfahnen. Die Reden von Joseph Goebbels und Hitler wurden im Rundfunk gesendet und in vielen Orten des Reiches auf öffentlichen Plätzen durch Lautsprecher übertragen - eine der ersten Erfahrungen medialer Simultanität in Deutschland. ${ }^{34}$

Hitler beschwor in seiner Rede die Einheit des deutschen Volkes, ohne die bestehenden Differenzen zu übergehen:

\begin{abstract}
Unseres Volkes Erwachen ist da. Das Symbol des Klassenkampfes, des ewigen Streites und Haders wandelt sich nun wieder zum Symbol der großen Einigung und Erhebung der Nation. Und deshalb haben wir diesen Tag der erwachenden Natur für alle kommenden Zeiten gewählt als Tag der Wiedergewinnung unserer eigenen Kraft und Stärke und damit auch zugleich jener schaffenden Arbeit, die keine engen Grenzen kennt, nicht gebunden ist an die Gewerkschaft, an Fabriken und Kontore, einer Arbeit, die wir überall dort anerkennen und fördern wollen, wo sie in gutem Sinne für Sein und Leben unseres Volkes geleistet wird. ${ }^{35}$
\end{abstract}

Die Vergangenheit malte Hitler in düsteren Farben. Klassenkampf, materielle und politische Not hätten zu Zerfall, Verzagtheit, Verzweiflung und zum Schwund der eigenen Kräfte geführt. Nun gelte es, den Weg zueinander wieder zu finden.

Wir wissen, dass dieser Prozess des Zueinanderfindens und gegenseitigen Verstehenlernens nicht eine Sache von Wochen oder Monaten, ja auch nur wenigen Jahren sein kann. Allein, wir haben den unerschütterlichen Willen, diese große Aufgabe vor der deutschen

33 Zum 1. Mai 1933 in Berlin siehe ausführlich: Eberhard Heuel, Der umworbene Stand. Die ideologische Integration der Arbeiter in den Nationalsozialismus 1933-1935, Frankfurt a.M./ New York 1988, S. 42-187; vgl. ebenfalls den Beitrag von Inge Marßolek in diesem Band.

34 Vgl.: Inge Marßolek, „Aus dem Volke, für das Volk“. Die Inszenierung der „Volksgemeinschaft" um und durch das Radio, in: dies./Adelheid von Saldern (Hrsg.), Radiozeiten. Herrschaft, Alltag, Gesellschaft (1924-1960), Potsdam 1999, S. 121-135.

35 Max Domarus, Hitler. Reden und Proklamationen 1932-1945. Kommentiert von einem Zeitgenossen, Teil I Triumph, Bd. I: 1932-1934, Leonberg 1988, S. 259. 
Geschichte zu erfüllen, haben den Entschluss, die deutschen Menschen wieder zueinander zu führen, und wenn es sein muss, zueinander zu zwingen. ${ }^{36}$

Kopf- und Handarbeiter sollten zueinander finden - und doch hob Hitler klar die manuelle Arbeit hervor. Das Vorurteil, dass Handarbeit minderwertig sei, müsse in Deutschland ausgerottet werden.

Wir wollen in einer Zeit, da Millionen unter uns leben ohne Verständnis für die Bedeutung des Handarbeitertums, das deutsche Volk durch die Arbeitsdienstpflicht zu der Erkenntnis erziehen, dass Handarbeit nicht schändet, nicht entehrt, sondern vielmehr wie jede andere Tätigkeit dem zur Ehre gereicht, der sie getreu und redlichen Sinnes erfüllt.“" ${ }^{\text {37 }}$ Stürmischen Beifall erhielt Hitlers Forderung: „Es bleibt unser unverrückbarer Entschluss, jeden einzelnen Deutschen, sei er, wer er sei, ob reich, ob arm, ob Sohn von Gelehrten oder Sohn von Fabrikarbeitern, einmal in seinem Leben zur Handarbeit zu führen, damit er sie kennenlernt. ${ }^{38}$

Am darauf folgenden Tag, dem 2. Mai 1933, besetzten SA-Stürme die Gewerkschaftshäuser, verhafteten Funktionäre und beschlagnahmten das Eigentum. An die Stelle der freien Gewerkschaften trat die Deutsche Arbeitsfront, die durch die Übernahme der Mitglieder wie des Vermögens der Gewerkschaften die mitgliederstärkste und reichste NS-Organisation wurde. In der Verordnung Hitlers über die Deutsche Arbeitsfront vom 24. Oktober 1934 hieß es unter dem § 2:

Das Ziel der Deutschen Arbeitsfront ist die Bildung einer wirklichen Volks- und Leistungsgemeinschaft aller Deutschen. Sie hat dafür zu sorgen, dass jeder einzelne seinen Platz im wirtschaftlichen Leben der Nation in der geistigen und körperlichen Verfassung einnehmen kann, die ihn zur höchsten Leistung befähigt und damit den größten Nutzen für die Volksgemeinschaft gewährleistet. ${ }^{39}$

\section{Arbeit als Dienst an der Volksgemeinschaft ${ }^{40}$}

Handarbeit als Erziehungsmittel - dieser Gedanke stand ebenso hinter der Institution des Reichsarbeitsdienstes wie dessen Funktion, junge arbeitslose Männer in öffentliche Arbeitsprogramme einzubinden. Kollektiv verrichtete Handarbeit als Medium von Vergemeinschaftung und Disziplinierung war das nationalsozi-

36 Ebd., S. 260.

37 Ebd., S. 262.

38 Ebd.

39 Zit. n.: Schneider, Unterm Hakenkreuz, S. 182.

40 Vgl. dazu auch den Beitrag von Harriet Scharnberg in diesem Band. 
alistische Konzept, das sich sowohl in den Lagern des Reichsarbeitsdienstes wie auch in den Konzentrationslagern wiederfindet. ${ }^{41}$

Zwar war der Reichsarbeitsdienst zunächst offiziell noch freiwillig und erst ab 1935 verpflichtend, aber mit einer Reihe von Maßnahmen und drohendem Entzug von Arbeitslosenunterstützung brachte man zahlreiche junge Männer dazu, sich für den RAD zu melden. ${ }^{42}$ Stärker als der ökonomische Nutzen und die statistische Verbesserung der Arbeitslosenzahl stand dabei die Formierungsabsicht im Vordergrund. „Der Reichsarbeitsdienst“, hieß es im Paragraph 3 des Gesetzes über den RAD, ,soll die deutsche Jugend im Geiste des Nationalsozialismus zur Volksgemeinschaft und zur wahren Arbeitsauffassung, vor allem zur gebührenden Achtung der Handarbeit erziehen.“43

In einem der ersten Interviews, die Hitler als neu ernannter Reichskanzler einer ausländischen Nachrichtenagentur gab, hatte er im Februar gegenüber Associated Press diese Aufgabe des Arbeitsdienstes unmissverständlich benannt:

Die Arbeitsdienstpflicht verdankt als Idee ihre Entstehung der katastrophalen wirtschaftlichen Not und der daraus entspringenden Arbeitslosigkeit. Die Arbeitsdienstpflicht soll vor allem verhindern, dass Hunderttausende unserer Jungarbeiter hilflos auf der Straße verkommen. Sie soll aber weiter durch eine allgemeine Erziehung zur Arbeit einer Überbrückung der Klassengegensätze dienen. Als Nationalsozialist sehe ich auch in der allgemeinen Arbeitsdienstpflicht ein Mittel, um zur Achtung vor der Arbeit zu erziehen. Unsere jungen Leute sollen lernen, dass die Arbeit den Menschen adelt. ${ }^{44}$

Und in einer Rede vor Autobahnarbeitern im September 1933 hob er noch einmal hervor,

dass wir einen Staat aufbauen wollen, der die Arbeit schätzt um ihrer selbst willen und der den Arbeiter achtet, weil er eine Pflicht an der Nation erfüllt, einen Staat, der durch seinen Arbeitsdienst jeden erziehen will, jedes Söhnchen auch hochgeborener Eltern zur Achtung der Arbeit, zum Respekt vor der körperlichen Tätigkeit im Dienste der Volksgemeinschaft. ${ }^{45}$

41 Kiran Patel, „Auslese“ und „Ausmerze“. Das Janusgesicht der nationalsozialistischen Lager, in: ZfG 54 (2006) 4, S. 339-365; Marc Buggeln/Michael Wildt, Lager im Nationalsozialismus. Gemeinschaft und Zwang, in: Bettina Greiner/Alan Kramer (Hrsg.), Die Welt der Lager. Zur „Erfolgsgeschichte“ einer Institution, Hamburg 2013, S. 217-253.

42 Detlev Humann, „Arbeitsschlacht“. Arbeitsbeschaffung und Propaganda in der NS-Zeit 1933-1939, Göttingen 2011, S. 365-480; Kiran Klaus Patel, „Soldaten der Arbeit“. Arbeitsdienste in Deutschland und den USA 1933-1945, Göttingen 2003.

43 RGBl. 1935 I, S. 769, zit. n. Patel, Soldaten der Arbeit, S. 107.

44 Domarus, Hitler. Reden und Proklamationen, Teil I, Bd. I, S. 212.

45 Ebd., S. 302. 
Dieser antibürgerliche Affekt gegen die „Hochgeborenen“, denen es gut tue, einmal körperlich zu arbeiten, wie es schon in der Rede zum 1. Mai 1933 explizit anklang, erschien auch später in Hitlers Reden, wie kurz vor Kriegsbeginn am 1. Mai 1939. Da gäbe es manche Volksgenossen, die glaubten, ihre Söhne seien $\mathrm{zu}$ etwas Besserem, Geistigem bestimmt und sollten daher von der Arbeitsdienstpflicht befreit werden.

\begin{abstract}
Was du, mein lieber Freund, schon unter Geist verstehst! Wenn dein Junge jetzt sechs Monate im Westen mit dem Spaten für Deutschland gearbeitet hat, dann hat er praktisch mehr geleistet, als vielleicht dein ganzer Geist Zeit seines Lebens für Deutschland leisten konnte. Und vor allem: Er hat ja mitgeholfen, eine der schlimmsten geistigen Verirrungen zu beseitigen, die es gibt, nämlich die innere Zerrissenheit eines Volkes. ${ }^{46}$
\end{abstract}

Auch von akademischer Seite erhielt diese Position prominente Unterstützung. Kein geringerer als der frisch gekürte Rektor der Universität Freiburg, der Philosoph Martin Heidegger, entfaltete in seiner Rektoratsrede vom 27. Mai 1933, welche Aufgaben die Universität zu erfüllen hätten:

Der Kampf, der heute tobt, geht um ganz große Ziele: eine Kultur kämpft um ihr Dasein, die Jahrtausende in sich verbindet und Griechen- und Germanentum gemeinsam umschließt. ${ }^{47}$

Wissenschaft „im Sinne des fragenden, ungedeckten Standhaltens inmitten der Ungewißheit des Seienden im Ganzen“ schaffe dem Volk seine wahrhaft geistige Welt.

Und die geistige Welt eines Volkes ist nicht der Überbau einer Kultur, sowenig wie das Zeughaus für verwendbare Kenntnisse und Werte, sondern sie ist die Macht der tiefsten Bewahrung seiner erd- und bluthaften Kräfte als Macht der innersten Erregung und weitesten Erschütterung seines Daseins. ${ }^{48}$

Für die Studenten hielt Heidegger drei „Bindungen“ parat: „Die erste Bindung ist die in der Volksgemeinschaft. Sie verpflichtet zum mittragenden und mithandelnden Teilhaben an Mühen, Trachten und Können aller Stände und Glieder des Volkes. Diese Bindung wird fortan festgemacht und in das studentische Dasein eingewurzelt durch den Arbeitsdienst.“49 Neben „Wehrdienst“ und

46 Rede Hitlers zum 1. Mai 1939, in: Max Domarus, Hitler. Reden und Proklamationen 19321945. Kommentiert von einem Zeitgenossen, Teil II Untergang, Bd. III: 1939-1940, Leonberg 1988, S. 1184.

47 Zit. n Victor Farías, Heidegger und der Nationalsozialismus, Frankfurt a.M. 1989, S. 155.

48 Zit. n.: ebd., S. 157.

49 Zit. n.: ebd., S. 159. 
„Wissensdienst“ galt die Arbeit als das wesentliche Element der Bindung und Teilhabe an der „Volksgemeinschaft“. In einer Ansprache wenige Monate später, im November 1934, anlässlich der feierlichen Immatrikulation wurde Heidegger unter der Überschrift „Der deutsche Student als Arbeiter“ noch deutlicher: „Der neue deutsche Student geht jetzt durch den Arbeitsdienst.“ Der wahre Sinn des „Wissensdienstes“ bestehe in der Eingliederung in die „Arbeitsfront", kurz:

Die Arbeit versetzt und fügt das Volk in das Wirkungsfeld aller wesentlichen Mächte des Seins. Das in der Arbeit und als Arbeit sich gestaltende Gefüge des völkischen Daseins ist der Staat. Der nationalsozialistische Staat ist der Arbeiterstaat. ${ }^{50}$

Iris Därmann machte mich darauf aufmerksam, wie eng Heideggers Verständnis von Gemeinschaft mit dem Krieg verbunden ist. In seinen beiden Vorträgen „Die deutsche Universität“ im August 1934 hieß es, dass die „eigentliche Vorbereitung der nationalsozialistischen Revolution“ im Ersten Weltkrieg begann, da im „Frontgeist“, begriffen vor allem als „Kameradschaft“, der „wissende Wille zu einer neuen Gemeinschaft“ entstehe. Der Kriegsdienst, der eine Todes- und Tötungsgemeinschaft begründet, bildete für Heidegger 1933/34 die eigentliche Gemeinschaftsform des Mitseins. Arbeit als Dienst an der Volksgemeinschaft umfasst auch „destruktive Arbeit“, „Vernichtungsarbeit“. ${ }^{1}$

\section{Arbeit und Gewalt}

Erziehung und Disziplinierung durch Arbeit stand auch bei den frühen Konzentrationslagern propagandistisch im Vordergrund. Für Reichsinnenminister Wilhelm Frick stand gleich Anfang März 1933, unmittelbar als nach dem Reichstagsbrand die massive Verfolgung von politischen Oppositionellen begann, fest, dass die Kommunisten nicht an der Eröffnung des neu gewählten Reichstages teilnehmen werden. „Diese Herrschaften“, so Frick „müssen wieder an fruchtbringende Arbeit gewöhnt werden. Dazu werden wir ihnen in Konzentrationslagern Gelegenheit geben. Wenn sie sich dann wieder zu nützlichen Mitgliedern der Nation erziehen lassen, wollen wir sie als vollwertige Volksgenossen will-

50 Ebd., S. 181.

51 Vgl.: Lars Clausen, Produktive Arbeit, destruktive Arbeit. Soziologische Grundlagen, Berlin/ New York 1988. 
kommen heißen, sonst werden wir sie auf die Dauer unschädlich zu machen wissen. “52

Was propagandistisch als „Erziehung“ deklariert wurde, diente im Alltag des Konzentrationslagers der Erniedrigung und Bestrafung. So berichtete die „Münchner Illustrierte Presse“ am 16. Juli 1933 in einem großen Artikel mit etlichen Fotografien über das Konzentrationslager Dachau und zeigte unter anderem ein Bild, auf dem mehrere Häftlinge eine Straßenwalze ziehen mussten. In der Bildunterschrift hieß es dazu:

Volksverführern, denen der Begriff der Arbeit ihr Leben lang fremd geblieben ist, lernen ihn hier zum eigenen Nutzen kennen. Zum ersten Mal arbeiten sie produktiv in einer Gemeinschaft. ${ }^{53}$

Das Arbeitskommando „Straßenwalze“, eine Straßenbaukolonne, dem Juden und so genannte „Bonzen“, also ehemalige sozialdemokratische Funktionären, angehörten, war berüchtigt wegen der Schwere und Brutalität der Arbeit.

„Vernichtung durch Arbeit“ - dieses absolut destruktive Verständnis von Arbeit kann wohl allein das NS-Regime für sich verbuchen. Dokumentiert ist diese Formulierung im Abkommen, dass Heinrich Himmler mit dem damaligen Justizminister Thierack im September 1942 schloss. Den Hintergrund bildete das Bemühen Himmlers, einen Teil der Strafrechtspflege, nämlich gegen die sogenannten „Fremdvölkischen“ von der Justiz an SS und Polizei zu übertragen. Thierack und Himmler kamen rasch überein, dass „,nicht genügende Justizurteile durch polizeiliche Sonderbehandlung [...] korrigiert“ werden sollten. Außerdem sollten sämtliche „asozialen Elemente“, wörtlich wurden genannt: „Juden, Zigeuner, Russen und Ukrainer, Polen über 3 Jahre Strafe, Tschechen oder Deutsche über 8 Jahren Strafe“, an die SS zwecks „Vernichtung durch Arbeit“ übergeben werden. ${ }^{54}$

Hitler dachte genauso. In den Tischgesprächen klagte er immer wieder über die Justiz, die nicht in der Lage sei, mit angeblich Kriminellen umzugehen. „Nach zehn Jahren Zuchthaus“, so Hitler im Februar 1942,

52 Wilhelm Frick im Völkischen Beobachter, Münchener Ausgabe, 11./12. März 1933, S. 2, zit. n.: Tuchel, Organisationsgeschichte, S. 44f.

53 Zit. n.: Hans-Günter Richardi, Schule der Gewalt. Das Konzentrationslager Dachau, München/Zürich 1995, S. 200.

54 BArch, R 22/4062, Bl. 35a-37, Vermerk Thieracks über die Besprechung am 18.9.1942, abgedruckt in: IMG, Bd. XXVI, S. 200-203 (654-PS); BA, R 22/5029, Bl. 58-65, Niederschrift Streckenbachs zu dem Treffen v. 21.9.1942. 
ist der Mensch sowieso für die Volksgemeinschaft verloren. Wer will ihm denn noch Arbeit geben? Solchen Kerl steckt man entweder in ein Konzentrationslager oder tötet ihn. In dieser Zeit ist das letztere wichtiger, um der Abschreckung willen. Um ein Exempel zu statuieren, muss es auch alle Mitläufer treffen! $!^{55}$

Auch in seiner rassistischen Definition von Arbeit blieb Hitler bei seinen Maximen, die er 1920 seinem Publikum verkündet hat. Im Oktober 1940 entwickelte er in einer Unterhaltung mit Generalgouverneur Hans Frank, wie Martin Bormann pflichteifrig mitstenographierte, seine Vorstellungen, wie mit den Polen umgegangen werden müsse:

Der Führer betonte weiter, der Pole sei im Gegensatz zu unserem deutschen Arbeiter geradezu zu niedriger Arbeit geboren. [...] Während unser deutscher Arbeiter von Natur aus im allgemeinen strebsam und fleißig sei, sei der Pole von Natur aus faul und müsse zur Arbeit angetrieben werden. [...] Es sei also durchaus richtig, wenn im Generalgouvernement eine starke Übersetzung an Arbeitskräften vorhanden sei, damit von dort aus wirklich alljährlich die notwendigen Arbeiter in das Reich kämen. - Unbedingt zu beachten sei, dass es keine ,polnischen Herren' geben dürfte: wo polnische Herren vorhanden seien, sollten sie, so hart das klingen möge, umgebracht werden. ${ }^{56}$

In einem Kommentar aus dem Reichsministerium für die besetzten Ostgebiete zum so genannten „Generalplan Ost“, der in der SS-Führung 1942 für die deutsche Besiedlung und Beherrschung Osteuropas, der Ukraine, des Baltikums, der Wolga-Region, der Krim und anderen Gebieten ausgearbeitet wurde und in dem mit der Vertreibung, Deportation und Ermordung von über 30 Millionen Menschen, die in diesen Gebieten lebten, geplant wurde, kam der Vorschlag auf, „ob nicht durch die Industrialisierung des baltischen Raumes zweckmäßigerweise die rassisch unerwünschten Teile der Bevölkerung verschrottet werden könnten“, d. h. durch Schwerstarbeit vernichtet werden sollten. ${ }^{57}$

Nur im Nationalsozialismus kam das Schlagwort von der „Vernichtung durch Arbeit“ auf, die Vorstellung, dass Menschen durch Arbeit „,verschrottet“ werden. Das rationale-zynische Kalkül, das auch westlichen Gesellschaften nicht fremd ist, durch Zwang und Unfreiheit Menschen zu ökonomisch günstigen Konditionen arbeiten zu lassen und dadurch den gesamtgesellschaftlichen

55 Henry Picker, Hitlers Tischgespräche im Führerhauptquartier. Entstehung, Struktur und Folgen des Nationalsozialismus, Frankfurt a.M./Berlin 1993, S. 143 (Eintrag vom 8.2.1942).

56 Martin Bormann, Aktenvermerk vom 2.10.1940, Nbg. Dok. USSR-172, dokumentiert in: Der Prozeß gegen die Hauptkriegsverbrecher vor dem Internationalen Militärgerichtshof, Nürnberg 14. November 1945 - 1. Oktober 1946, Nürnberg 1947, Bd. VII, S. 252-255, Zitat: S. $252 f$.

57 Bericht Wetzels über die Besprechung im Ostministerium am 4.2.1942 (Nbg. Dok. NO-2585), abgedruckt in: Helmut Heiber, Der Generalplan Ost, in: VfZ 6 (1958), S. 281-325, hier S. 293-296. 
Nutzen bzw. den Kapitalgewinn für einige Gruppen oder Unternehmen zu steigern, stand im Nationalsozialismus hinter rassistischen, antisemitischen Weltordnungsplänen zurück. Auch wenn das NS-Regime immer wieder zu pragmatischen, der Kriegssituation geschuldeten Entscheidungen gezwungen war. An der rassistischen Grundhaltung, dass Juden ebenso wenig eine Existenzberechtigung haben wie behinderte Menschen, änderte das nichts.

\section{Schluss}

Was Hitlers Arbeitsbegriff schon 1920 auszeichnete, war die rassistische und antisemitische Grundierung. Während „Arier“ durch die unwirtlichen Umstände ihrer Umgebung zu kulturschaffender Arbeit erzogen worden seien, seien „Juden“ hingegen zu einer gemeinnützigen Arbeit nicht fähig. Diese durchaus in völkischer Tradition stehende Wendung erlaubte es zum einen, die moderne kapitalistische Gesellschaft zu kritisieren, in deren Mittelpunkt das Einzelinteresse stehe. Zum anderen ließen sich eben „raffende“ Kapitalisten als ,jüdisch“ denunzieren, die gegen das Gemeinwohl arbeiten würden. Zudem bot die grundlegende Annahme, dass allein die Arbeit Wert schaffe, Berührungspunkte mit der sozialistischen Arbeiterbewegung. Dass auch die Sozialdemokraten mit Antisemitismus, der Denunziation des „jüdischen Kapitals“ zu kämpfen hatten, zeigt deren Geschichte immer wieder.

Hitlers Rede von der Arbeit als Dienst an der Volksgemeinschaft, von der Handarbeit, die als erzieherische Maßnahme gerade den „Tintenrittern“ und „hochwohlgeborenen Söhnchen“ zugutekomme, wie sie insbesondere in seiner Ansprache zum 1. Mai 1933 zum Ausdruck kommt, konnte auf ein breites Einverständnis, auch über das völkische Lager hinaus, rechnen. Auch die Vorstellung, Arbeit als harte Erziehungsmaßnahme und Strafe einzusetzen, hatte Traditionen, die weit vor dem 20. Jahrhundert lagen.

Was die nationalsozialistische Arbeitsauffassung heraushob, war ihre Destruktivität. In den Äußerungen Hitlers zur Arbeit, die Polen zu verrichten hätten, ist der gewalttätige, rücksichtslose Rassismus ganz unverhüllt, der - anders als die Legitimationen der europäischen Kolonisatoren - keinerlei Anspruch auf eine „Zivilisierungsmission“ mehr erhob. ${ }^{58}$ Hier sollten Menschen

58 Vgl. dazu: Felix Axster, Arbeit, Teilhabe und Ausschluss. Zum Verhältnis zwischen kolonialem Rassismus und nationalsozialistischem Antisemitismus, in: Birthe Kundrus/Sybille Steinbacher (Hrsg.): Kontinuitäten und Diskontinuitäten. Der Nationalsozialismus in der Geschichte des 20. Jahrhunderts, Göttingen 2013, S. 121-133. 
bis zu ihrer „Verschrottung“ für das Deutsche Reich arbeiten, allein von einem utilitaristischen Kalkül ausgehend, das nicht einmal mehr die „Reproduktionsfähigkeit“ der Arbeitskräfte zu berücksichtigen glaubte. Hitlers grundlegende Überzeugungen zur Arbeit, wie er sie insbesondere in seinen Reden vom 20. August 1920 und 1. Mai 1933 entwickelt hatte, waren erkennbar auch die Richtschnur für nationalsozialistisches Handeln zwischen 1933 und 1945. 